\title{
Nanofluidic Pulser Based on Polymer Conical Nanopores
}

\author{
Lin Wang, ${ }^{\dagger}$ Lixin Sun, ${ }^{\dagger}$ Ceming Wang, ${ }^{\dagger}$ Long Chen, ${ }^{\dagger}$ Liuxuan Cao, ${ }^{\dagger}$ Guoqing $\mathrm{Hu}^{\neq}{ }^{\dagger}$ Jianming Xue, ${ }^{*}{ }^{\dagger}$ and \\ Yugang Wang ${ }^{+}$
}
†State Key Laboratory of Nuclear Physics and Technology, School of Physics, and Center for Applied Physics and Technology, Peking University, Beijing 100871, People's Republic of China
${ }^{\ddagger}$ State Key Laboratory of Nonlinear Mechanics, Institute of Mechanics, Chinese Academy of Sciences, Beijing 100190, People's Republic of China

Supporting Information

ABSTRACT: The study of voltage-dependent ion current fluctuation phenomena in synthetic nanopores is important as it is helpful to investigate the mechanism of mass transport in nanoscale systems, which have similarities with natural ion channels in the biological cell membrane. Moreover, we could fabricate some high-performance nanofluidic devices through clearly understanding ion current fluctuation behavior. In this paper, we report a nanofluidic pulser induced by formation and dissolution of weakly soluble salts in conical nanopores. The

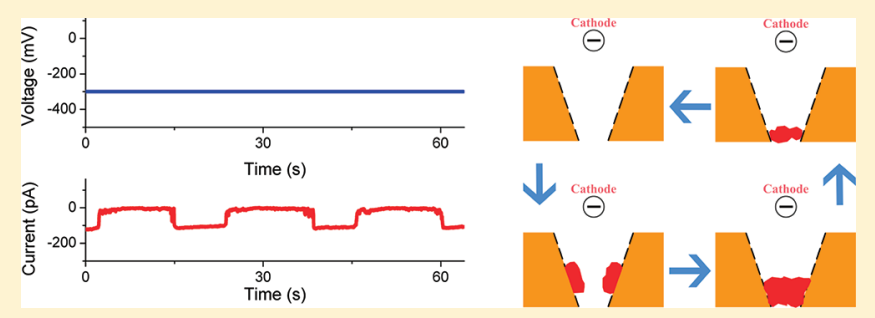
current fluctuation signals are easily controllable in $1 \mathrm{M} \mathrm{KCl}$ electrolyte. Amplitude, frequency, and waveform of ion fluctuation current of the nanofluidic pulser could be controlled by changing the applied negative voltage, and the time ratio of pore opening/ closing could be simply manipulated by the concentration of the bivalent cation. A high-quality square wave of ion current signal is found, especially when the negative voltage is below $300 \mathrm{mV}$. Additionally, we developed a new model about the formation and dissolution process of precipitation. Our work is helpful for the design of nanoscale ion current waveform generators in the future.

\section{INTRODUCTION}

Single nanopores have attracted considerable interest for their novel applications in chemical and biological analysis. Nanoporebased devices are emerging as important tools for detection and analysis of various biomolecules, mainly by monitoring the flow of ions through a narrow pore and detecting changes in ion current as molecules pass through this pore. ${ }^{1-6}$ With the recent development of nanofabrication techniques, nanopores made of polymer materials, silicon, or silicon-containing compounds have begun to replace the original biological nanopores because of their better long-term stability. ${ }^{7,8}$ These artificial nanopores immersed in electrolyte solution are also a convenient model to examine the processes that happen within natural ion channels, both sharing two main functional characteristics: ion selectivity and response to an external electric field. ${ }^{9-11}$

It has been demonstrated that current fluctuations, interpreted as "open-closed" behavior, typical of that observed for a patch-clamped single ion channel may be reproduced by a voltage-clamped narrow poly(ethylene terephthalate) (PET) pore even without lipid and protein. ${ }^{12}$ Korchev et al. ${ }^{13}$ also observed fluctuations of ion selectivity and ion current through both PET pores and toxin-induced pores in a lipid bilayer. They concluded that these fluctuations are likely due to the same cause: a fluctuation of surface charge of the pores. Recently, several other groups investigated the ion current fluctuations through the solid-state nanopores and classified the noise sources into a high-frequency noise and a low-frequency noise. ${ }^{14,15}$ Tuning the geometry and surface chemistry of nanopores helps create devices that control transport of ions and molecules in solution. One example is the asymmetric conically shaped nanpores in which the current-voltage curves show non-Ohmic behavior of current rectifying. ${ }^{16}$ In a conical nanopore, the negative surface charge results in the accumulation of counterions and the exclusion of co-ions near the surfaces, whereas the negative applied voltage accords with the change in ion concentrations inside the nanopore. It has been found that ionic current through a charged conical nanopore could oscillate in the presence of weakly soluble salts such as calcium ions. ${ }^{17}$ Initially they explained the fluctuations as results of transient binding of calcium ions to carboxyl groups on the pore walls. Lately, Powell et al. ${ }^{18}$ discovered that the oscillating ionic current is caused by the transient formation and redissolution of nanoprecipitates. Once the ion concentration of weakly soluble salts exceeds the level of solubility product, nanoprecipitates form and block the ion current passing through the nanopore, resulting in voltage-dependent current fluctuations. These ionic current fluctuations in nanopores have great potential in applications of stochastic sensors, kinetics studies of crystallization, and nanoscale ion current waveform generators.

\footnotetext{
Received: $\quad$ May 21, 2011

Revised: September 18, 2011

Published: October 20, 2011
} 
Though previous investigations have demonstrated that precipitation-induced current fluctuations can be influenced by many system parameters such as applied voltages, concentration of divalent ions, and electrolyte buffers, ${ }^{18,19}$ a further study still needs to be performed to examine such fluctuation behaviors efficiently and to elucidate the mechanisms of formation and dissolution of precipitation. Moreover, how to precisely control the ionic fluctuation current waveform is still a great challenge for handpicked designing of special current signal generators. In this paper, we report a nanofluidic pulser based on the precipitationinduced ionic current fluctuations in conical nanopores. A highquality pulser can be obtained under negative applied voltages in a high-concentration $\mathrm{KCl}$ buffer solution, with its amplitude, frequency, waveform, and the opening/closing ratio being manipulated by adjusting the operating parameters. In addition, we develop a new theoretical model on the migration of surface charge screened region to explain the process of ionic current fluctuation, for which the tendency of ion current fits well with the experimental data.

\section{EXPERIMENTAL METHODS}

2.1. Fabrication of Single Conical Nanopores. The single nanopores presented in this paper were prepared with chemical etching technique in $12 \mu \mathrm{m}$ thick foils of poly(ethylene terephthalate) (PET). ${ }^{20,21}$ Briefly, the foils were irradiated first with single swift heavy ions and subsequently processed with an asymmetric chemical etching method. The details of conical nanopore preparation are available in Supporting Information. For a conical nanopore, the large opening of the nanopore is called the base (with diameter $D_{\text {base }}$ ) while the small opening is called the tip (with diameter $D_{\text {tip }}$ ).

2.2. Ion Current Recording. A single conical nanopore membrane was mounted into a pair of conductivity cells. $\mathrm{Ag} / \mathrm{AgCl}$ electrodes were inserted into each half-cell solution for applying the transmembrane potential and measuring the ion current. The base opening side was contacted with applied voltage, while the tip opening side was contacted with ground (schematic diagram is shown in Supporting Information). The ion currents were recorded with an Axon Axopatch 200B amplifier (Molecular Devices Inc.) in voltage-clamp mode, using $10 \mathrm{kHz}$ sampling frequency and low-pass Bessel filters from $100 \mathrm{~Hz}$ to $2 \mathrm{kHz}$. The signal was digitized with Digidata 1322A (Molecular Devices Inc.) and the recordings of ion current in time were analyzed by use of the commercial software Clampfit 9.0 (Molecular Devices Inc.). The range of scanning voltage was mainly from $-1 \mathrm{~V}$ to $+1 \mathrm{~V}$. The series of potassium chloride solutions with different concentrations of $\mathrm{CaCl}_{2}$ or $\mathrm{CoCl}_{2}$ and phosphate buffer (PBS) were prepared from commercial chemical agents with Milli-Q water (18.2 M $\Omega$ ).

\section{RESULTS AND DISCUSSION}

3.1. Experimental Results. The main purpose of the present study is to create a tunable nanofluidic pulser by seeking suitable ionic solution conditions. In previous work, the working electrolyte was $0.1 \mathrm{M} \mathrm{KCl}$ buffer solution. Most of the current fluctuation signals were transient pulses and the control of open/closed state ratio was inconspicuous. We found that properly increasing the concentration of working electrolyte would result in better current fluctuation signals. Moreover, under low negative applied voltages, different fluctuation
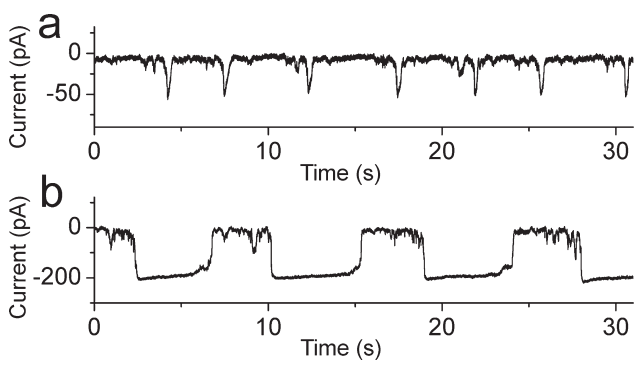

Figure 1. Different ion current fluctuation behaviors of a single conical nanopore $\left(D_{\text {tip }}=5 \mathrm{~nm}\right)$ in various concentrations of $\mathrm{KCl}$ buffer under low negative voltage. (a) Ion current in time recorded at $-300 \mathrm{mV}$ in a solution of $0.1 \mathrm{M} \mathrm{KCl}, 0.2 \mathrm{mM} \mathrm{CoCl}_{2}$, and $2 \mathrm{mM}$ PBS. (b) Ion current in time recorded at $-300 \mathrm{mV}$ in a solution of $1 \mathrm{M} \mathrm{KCl}, 0.5 \mathrm{mM} \mathrm{CoCl}_{2}$, and $4 \mathrm{mM}$ PBS.

patterns appeared after $0.1 \mathrm{M} \mathrm{KCl}$ solution was replaced by $1 \mathrm{M} \mathrm{KCl}$ solution.

Figure 1 shows the discrepancy of ion current fluctuation behaviors in a $5 \mathrm{~nm}$ PET nanopore in $0.1 \mathrm{M} \mathrm{KCl}$ and $1 \mathrm{M} \mathrm{KCl}$ solutions, whereas the concentrations of additional $\mathrm{CoCl}_{2}$ and PBS are both at millimolar levels. The ion current signals of open state change their waveforms from a pulse shape to a rectangle shape under low negative voltage. Though the concentrations of $\mathrm{CoCl}_{2}$ and PBS were not identical in the buffer solutions, this trend was found in most of the cases in our experiments. The rectangular-shape current signals are of interest because the steady signals of two different conductance states indicate our nanopore is more like a controllable ion current switch. Moreover, the rectangular shape current resembles those due to the rapid process of current changes in biological ion channels.

A single conical nanopore with a tip diameter of $3.5 \mathrm{~nm}$ was systematically measured in $1 \mathrm{M} \mathrm{KCl}$ solution with $0.8 \mathrm{mM} \mathrm{CoCl}_{2}$ and $4 \mathrm{mM}$ PBS (at $\mathrm{pH}$ ). The ionic current fluctuations were observed only under negative applied voltages, and current signals as a function of elapsed time are shown in Figure 2. The amplitudes of ionic current signals are voltage-responsive: a higher negative voltage causes a greater amplitude current fluctuation. It is interesting to note that the current fluctuation is more regular and stable when the applied negative voltage is below $500 \mathrm{mV}$. Meanwhile, the dissociation degree of current amplitudes becomes large when high negative voltages are applied (see -1000 and $-800 \mathrm{mV}$ in Figure 2a,b).

Shapes of ion current signals can also be affected by applied voltages. Open state of the nanopore is presented as pulse waveform under $-1000 \mathrm{mV}$. As the voltage decreases, fluctuation current gradually shows a quick pore-opening process and a slow pore-closing process. Hence we could easily observe the asymmetric shape waveform of current signals at $-500 \mathrm{mV}$. When the negative voltage is below $300 \mathrm{mV}$, both ion currents in open state and closed state show their stabilities at most duration times, and the transitions of pore opening and pore closing are transient compared with the whole oscillation cycle. Additionally, a special plateau current in open state could be found, especially when the voltage is $-200 \mathrm{mV}$.

From Figure 2 we can see that the applied voltage also has an impact on the frequency of current signals. The period of fluctuation events increases with the drop of the applied voltage. After statistically analyzing the data (statistic rules are available in Supporting Information), we found that the fluctuation events within $1 \mathrm{~min}$ decreased from 46 to 5 as the voltage changed 

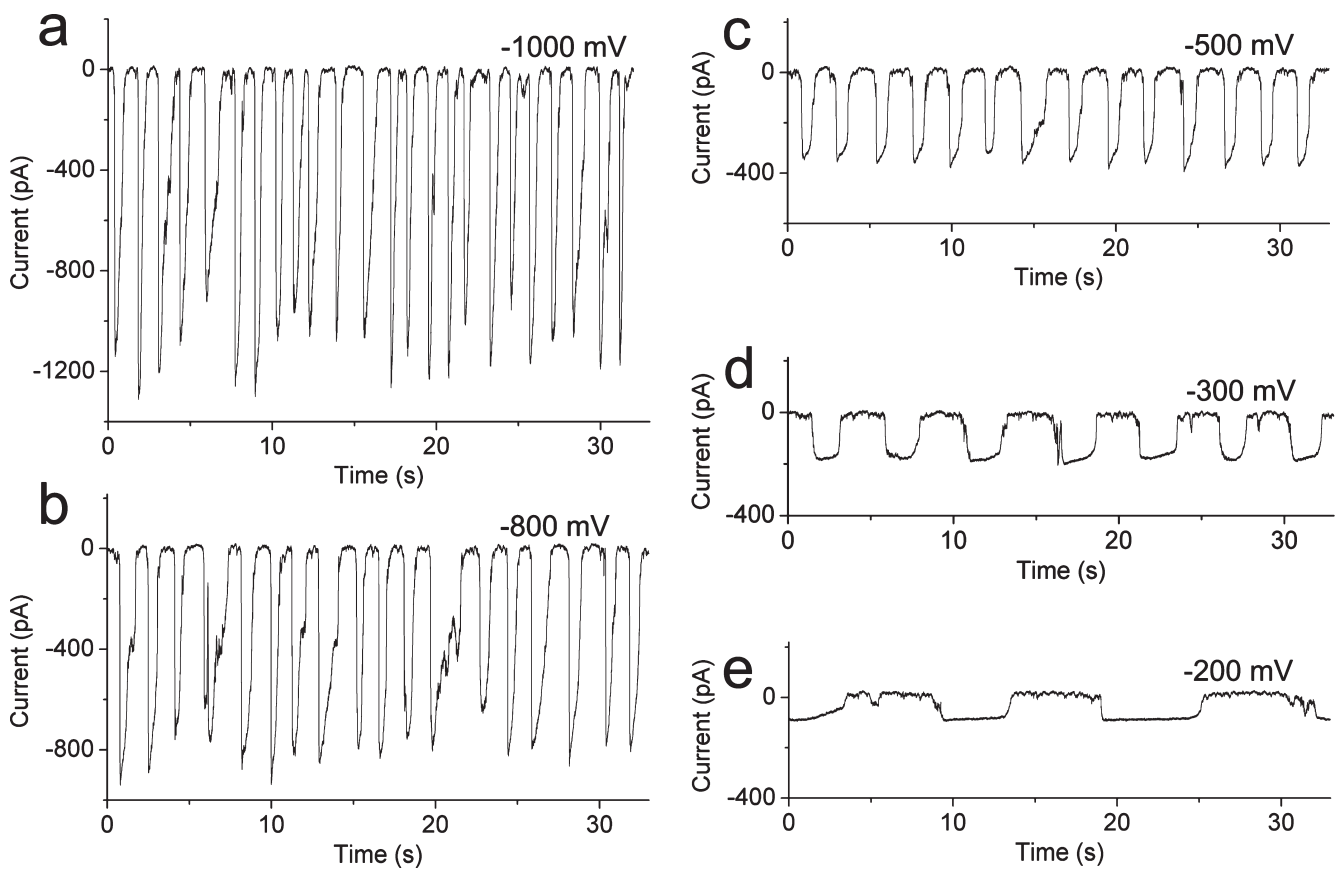

Figure 2. Transport properties of a single conical nanopore with a diameter of $3.5 \mathrm{~nm}$ studied in $1 \mathrm{M} \mathrm{KCl}, 0.8 \mathrm{mM} \mathrm{CoCl}_{2}$, and $4 \mathrm{mM} \mathrm{PBS}$. Examples of ion current signals in time recorded at (a) $-1000,(\mathrm{~b})-800,(\mathrm{c})-500,(\mathrm{~d})-300$, and (e) $-200 \mathrm{mV}$ are shown.

from -1000 to $-200 \mathrm{mV}$ (Figure 3a). This approximately linear relationship indicates that the formation of $\mathrm{CoHPO}_{4}$ precipitation slows down under low voltages.

Figure $3 \mathrm{~b}$ shows the average duration time of pore open and closed states. Both of them present an exponentially increasing relationship with decreasing voltage. Duration time of closed state is comparable with that of open state under every certain voltage from -200 to $-1000 \mathrm{mV}$. The corresponding time ratios of pore open and closed states in the average oscillation cycle are also calculated in Figure 3c, and results show both of them are around 0.5 . This might suggest that, as long as the fluctuation starts, the applied voltages have no significant influence on the ratio of pore opening or closing in $1 \mathrm{M} \mathrm{KCl}$ solution with $\mathrm{CoHPO}_{4}$ precipitation.

Controlling the time ratio of two states in an oscillation cycle is crucial in the applications of high-quality square waveforms of ion current signals. The time ratio is a manifestation of the competition between formation and dissolution processes of the precipitation, and we are able to tune this ratio by just simply changing the concentrations of $\mathrm{CoCl}_{2}$. The $3.5 \mathrm{~nm}$ nanopore was subsequently used to record ion currents in $1 \mathrm{M} \mathrm{KCl}$ and $4 \mathrm{mM}$ $\mathrm{PBS}$ with various concentrations of $\mathrm{CoCl}_{2}$ at $-200 \mathrm{mV}$. With the addition of $0.4 \mathrm{mM} \mathrm{CoCl}_{2}$, the closed state is unstable and the pore-opening process is dominant (Figure $4 \mathrm{a}$ ), whereas the pore open and closed states keep balanced with $0.8 \mathrm{mM} \mathrm{CoCl}$ (Figure 4b). The fluctuation behavior would be mainly dominated by pore closed state as the concentration of $\mathrm{CoCl}_{2}$ further increases to $2 \mathrm{mM}$ (Figure 4c). As the increase in Co ions speeds up the formation of $\mathrm{CoHPO}_{4}$ precipitation, the time ratio of closed state dramatically increases from 0.17 to 0.85 as $\mathrm{CoCl}_{2}$ concentration changes from 0.4 to $2 \mathrm{mM}$.

We also investigated the influence of different ionic species on the fluctuation of current signals. The same conical nanopore was studied in $1 \mathrm{M} \mathrm{KCl}$ with $4 \mathrm{mM} \mathrm{CaCl}_{2}$ and $6 \mathrm{mM}$ PBS. Generally, the periodicity of $\mathrm{CaHPO}_{4}$-induced fluctuations is not as good as that of $\mathrm{CoHPO}_{4}$ due to the fast transition process between open state and closed state, as demonstrated in the previous study. ${ }^{19}$ However, by using $1 \mathrm{M} \mathrm{KCl}$ electrolyte, the special stable rectangular shape of ion current signals for $\mathrm{CaHPO}_{4}$ precipitation was also observed in our experiments under low negative voltages (Figure 5). See Supporting Information for more details.

3.2. Slowing Down of Nanoprecipitation Formation in High-Concentration $\mathrm{KCl}$ Solution. According to the experimental results mentioned above, the quality of current signals produced by $1 \mathrm{M} \mathrm{KCl}$ is significantly improved compared with the ones produced by $0.1 \mathrm{M} \mathrm{KCl}$ in previous studies. This indicates that a higher concentration of electrolyte solution is beneficial for obtaining and controlling better fluctuation current signals. We attribute this property to the slowing down of nanoprecipitation formation in $1 \mathrm{M} \mathrm{KCl}$.

First of all, the increase of $\mathrm{K}^{+}$and $\mathrm{Cl}^{-}$densities leads to a stronger counterion atmosphere surrounding $\mathrm{Co}^{2+}$ and $\mathrm{HPO}_{4}{ }^{2-}$ ions. For fixed concentration values of $\mathrm{CoCl}_{2}$ and $\mathrm{PBS}$, the binding probability between $\mathrm{Co}^{2+}$ and $\mathrm{HPO}_{4}{ }^{2-}$ becomes weaker; that is, the activity coefficients of $\mathrm{Co}^{2+}$ and $\mathrm{HPO}_{4}{ }^{2-}$ ions in $1 \mathrm{M} \mathrm{KCl}$ declined in comparison with $0.1 \mathrm{M} \mathrm{KCl}$. Hence a higher concentration of $\mathrm{Co}^{2+}$ might be required to start fluctuations of ion current. Our experimental results support this: the $3.5 \mathrm{~nm}$ nanopore could easily trigger the normal ion current fluctuation behaviors in $0.1 \mathrm{M} \mathrm{KCl}$ with the presence of $0.2 \mathrm{mM} \mathrm{CoCl}_{2}$ and $2 \mathrm{mM}$ PBS. However, if we fix the concentration of $\mathrm{CoCl}_{2}$ and $\mathrm{PBS}$ while changing the concentration of $\mathrm{KCl}$ from 0.1 to $1 \mathrm{M}$, the fluctuation current signals cannot be triggered even at $-1 \mathrm{~V}$. Moreover, as the increase of $\mathrm{K}^{+}$and $\mathrm{Cl}^{-}$densities leads to a thinner electrical double layer (EDL), the influence region of electrostatic interaction for surface charge on the pore wall is reduced. This indicates that the effect of enrichment for divalent ions in conical nanopore becomes weaker under high-concentration $\mathrm{KCl}$.

For the two reasons mentioned above, in a precipitationinduced nanopore system, a higher $\mathrm{KCl}$ concentration results in a 


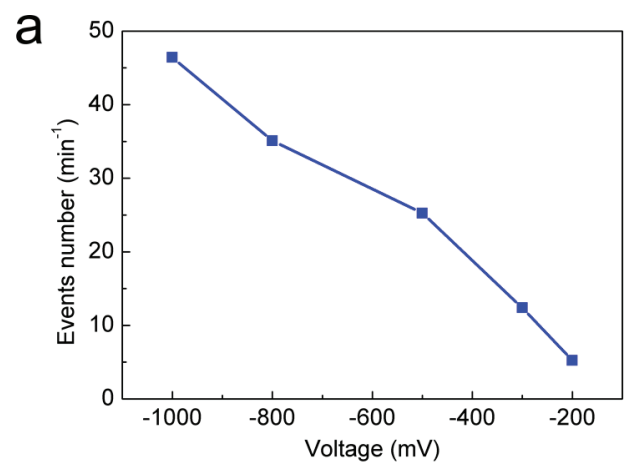

b
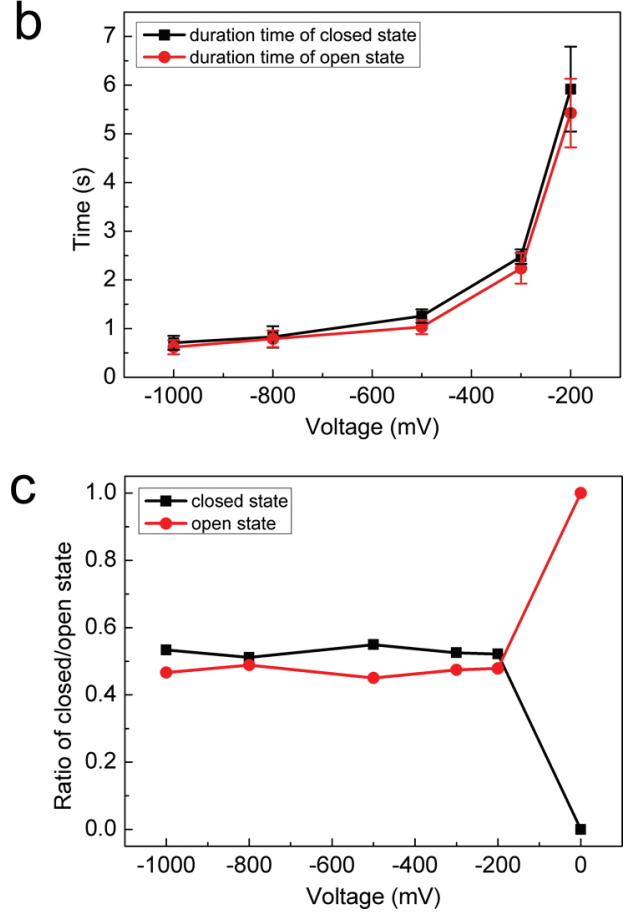

Figure 3. Statistical results of ion current signals about the $3.5 \mathrm{~nm}$ nanopore in the presence of $\mathrm{CoHPO}_{4}$. (a) Numbers of fluctuation events per minute as a function of voltage. (b) Duration times of pore open and closed states as a function of voltage. (c) Corresponding time ratio of pore open and closed state in the average oscillation cycle under various negative voltages.

slower process of precipitation formation, and pore closing cannot occur until enough precipitation is accumulated inside the nanopore. Because the dissolution process of precipitation becomes relatively sufficient in this situation, the overlap of ion current signals for adjacent oscillation cycles can be partly averted. Another crucial factor to induce degree of enrichment is applied negative voltages in the experiments: a lower voltage accords with a weaker degree of enrichment. So decreasing the voltage is a better choice to obtain the rectangle shape of ion current even under the situation of $1 \mathrm{KCl}$ solution. The above mechanism enables us to get long-period, high-quality, and stable fluctuation current signals in both closed and open states of the pore under low negative voltage in high-concentration $\mathrm{KCl}$ solution.

3.3. Theoretical Model for Ion Current Fluctuation. The discrete ion current under constant applied voltage indicates the different conductance states of the nanopore. This switching of various conductance states might result from the instability of the
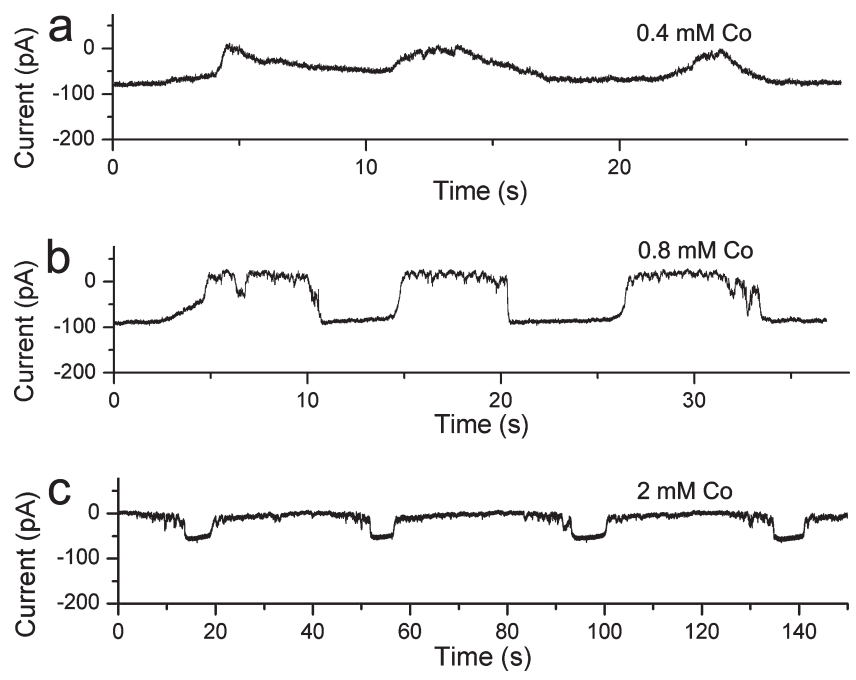

Figure 4. Transport properties of the same $3.5 \mathrm{~nm}$ single conical nanopore in $1 \mathrm{M} \mathrm{KCl}$ and $4 \mathrm{mM}$ PBS with various concentrations of $\mathrm{CoCl}_{2}$. Ion current signals in time were recorded when the concentrations of $\mathrm{CoCl}_{2}$ are (a) 0.4, (b) 0.8 , and (c) $2 \mathrm{mM}$.
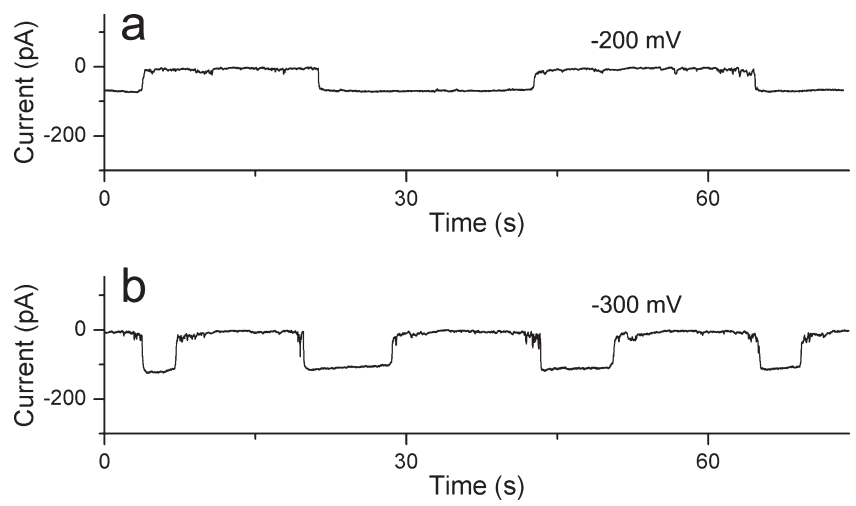

Figure 5. Transport properties of a single conical nanopore studied in $1 \mathrm{M} \mathrm{KCl}, 4 \mathrm{mM} \mathrm{CaCl}_{2}$, and $6 \mathrm{mM} \mathrm{PBS}$. Ion current signals in time were recorded at (a) -200 and (b) $-300 \mathrm{mV}$.

charged pore wall or the steric hindrance when large molecules pass through the nanopore. We believe that the precipitationinduced ion current fluctuations have a direct relationship with both factors.

Charged pore walls inside a nanopore have a great enrichment effect for divalent ions, and previous study has shown that the concentration of $\mathrm{Ca}^{2+}$ has a 2 orders of magnitude increase under $-1 \mathrm{~V}$ at the tip side of a track-etched conical nanopore. ${ }^{19}$ Nanoprecipitations would first form at the region close to the pore wall, where the enrichment effect is strongest. As precipitations are electroneutral, the negative surface charge is screened and the ion concentration distribution inside the nanopore is changed. Ion concentration decreases at the side of precipitation, which is close to the base opening of the nanopore, while increasing at the other side. Hence we assume that the zero surface charge region covered with precipitations might migrate inside the nanopore, and this process corresponds with dissolution of precipitations at the side close to base opening and forming of precipitation at the side close to tip opening of the nanopore. Next we analyzed the ion transport behaviors of the 

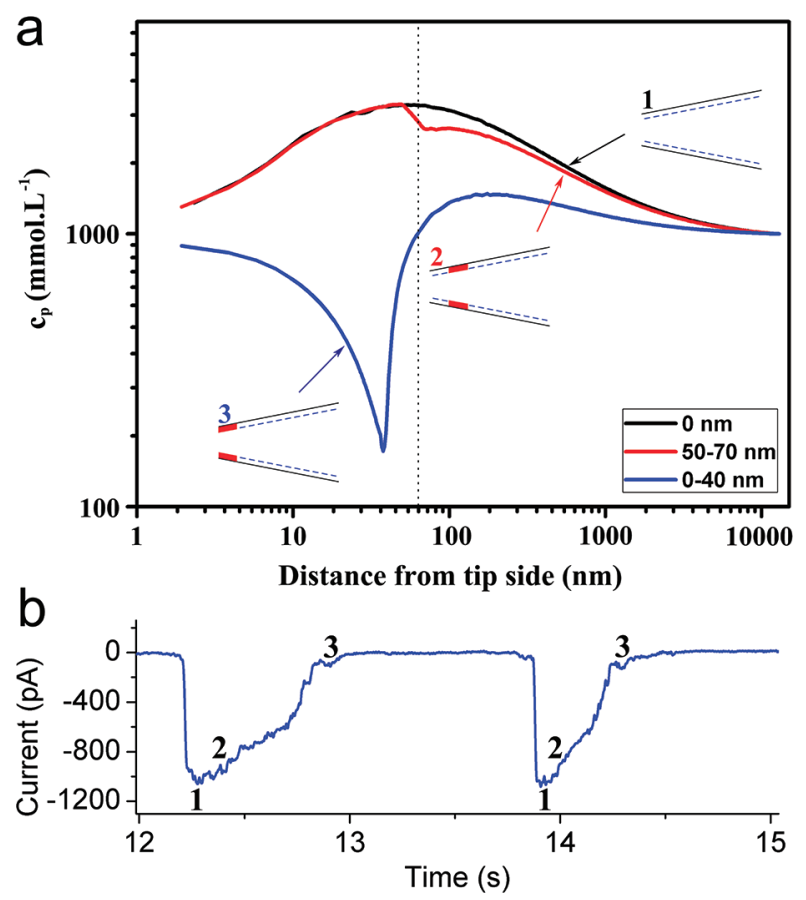

Figure 6. Calculated ion currents with various precipitation-covered regions show similarity to precipitation-induced fluctuation current behavior. (a) Changes of potassium ion concentration distribution along the axial direction of nanopore with migration of the precipitationcovered region. (b) Ion current signals of $3.5 \mathrm{~nm}$ nanopore when the applied voltage is $-1 \mathrm{~V}$ and the electrolyte is $1 \mathrm{M} \mathrm{KCl}$.

migration of precipitation-covered region by numerically solving Poisson-Nernst-Planck (PNP) equations. The method of theoretical calculation can be found in our previous work ${ }^{22}$ and in Supporting Information.

In order to simplify calculations, we made three assumptions: (1) the region covered with precipitation of the pore wall is migratory; (2) precipitation has the property of electric neutrality and plays the role of screening surface charge; and (3) thickness of precipitation is neglected. The calculation parameters we used are as follows: $D_{\text {tip }}=3.5 \mathrm{~nm}, D_{\text {base }}=500 \mathrm{~nm}$, and surface charge densities are $-0.08 \mathrm{C} / \mathrm{m}^{2}$ for normal pore walls and $0 \mathrm{C} / \mathrm{m}^{2}$ for the precipitation-covered regions. The electrolyte solutions are $1 \mathrm{M} \mathrm{KCl}$ with millimolar concentrations of $\mathrm{CoCl}_{2}$ or $\mathrm{CaCl}_{2}$ and PBS. As the concentrations of potassium ions and chlorine ions are higher than those of calcium ions, cobalt ions, and PBS, we neglect the contribution of these weakly soluble salts to the ion current.

Figure 6a is a plot of potassium ion concentration $\left(c_{\mathrm{p}}\right)$ along the axial direction of the nanopore when the nanoprecipitationcovered region migrates regularly. When there is no precipitation on the pore wall, the strongest enrichment region is about $60 \mathrm{~nm}$ from the tip side of the nanopore (state 1 in Figure 6a). Because nanoprecipitations first form at the region where the enrichment effect is strongest, we assume the precipitation-covered region is $50-70 \mathrm{~nm}$ from tip side of the nanopore. We also calculated $c_{\mathrm{p}}$ based on the $20 \mathrm{~nm}$ length precipitation. The calculation results show that $c_{\mathrm{p}}$ does not have an obvious change at the location of $50 \mathrm{~nm}$ but had a large drop at the location of $70 \mathrm{~nm}$ (state 2 in Figure 6a). This result indicates that precipitations might continuously form at the side close to tip opening of the nanopore and dissolve at the side close to base opening of the nanopore.
When the precipitation-covered region migrates to $0-40 \mathrm{~nm}, c_{\mathrm{p}}$ drops to a very low level in the whole nanopore (state 3 in Figure 6a). In this case, the dissolution of precipitation from base side of the nanopore is dominant and $c_{p}$ starts to increase gradually until the precipitation-covered region disappears.

The calculated ion currents from states 1-3 (Figure 6a) are $-3.7,-3.5$, and $-1.2 \mathrm{nA}$, respectively. The migration of zero surface charge regions could cause a 70\% drop of ion current, and the formation and dissolution processes of precipitation accord with the trend of decreasing first and then increasing for absolute value of ion current. This tendency of the calculated results agrees with the current fluctuation behaviors in the experiments. The changes of states $1-3$ in Figure 6a correspond to the changes of states $1-3$ in ion current recording signals in real time (Figure 6b). However, there is a discrepancy of ion current between calculated results and experimental results. This discrepancy might come from neglect of the thickness of precipitation on the pore wall. For example, when the region of $30-70 \mathrm{~nm}$ from the tip opening is covered with $1 \mathrm{~nm}$ thickness of precipitation, ion current changes from -3.07 to $-1.72 \mathrm{nA}$. And when the region extends to $0-70 \mathrm{~nm}$, the ion current changes from -1 to $-0.48 \mathrm{nA}$. However, the ion current will not become zero as long as the pore is in open state. Hence we presume that precipitation-induced ion current fluctuation behaviors might be a synergistic result of both migration of precipitation-covered region and volumetric block of precipitation inside nanopores.

\section{CONCLUSION}

In summary, we found a controllable nanofluidic pulser based on precipitation-induced ion current fluctuations inside nanopores in $1 \mathrm{M} \mathrm{KCl}$ electrolyte solution. By manipulating applied voltages, we are able to control the amplitude, frequency, and waveform of fluctuation current signals of the pulser. The ratio of pore opening is also tunable by changing the concentration of bivalent cations. High-quality square current signal is found, especially when the negative voltage is below $300 \mathrm{mV}$. We attribute this well-controlled fluctuation pattern to the slowing down of nanoprecipitation formation in high-concentration $\mathrm{KCl}$ solution. In addition, we developed a new model with theoretical calculations to propose a mechanism that both migration of precipitation-covered region and volumetric block of nanoprecipitation inside nanopores result in ion current fluctuation signals. Our work is helpful for the design of high-quality nanoscale ion current pulse signal generators.

\section{ASSOCIATED CONTENT}

S Supporting Information. Additional text and five figures showing fabrication of single conical nanopores, analysis rules for ion current signals, ion current fluctuation behavior in the presence of $\mathrm{CaHPO}_{4}$, and theoretical calculation based on Poisson-Nernst-Planck equations. This material is available free of charge via the Internet at http://pubs.acs.org.

\section{AUTHOR INFORMATION}

\section{Corresponding Author}

*E-mail jmxue@pku.edu.cn; tel 86-10-62758494; fax 86-1062751875 . 


\section{ACKNOWLEDGMENT}

Financial support from NSFC (Grants 10975009 and 90923004) to J.X. and National 973 Project (Grant 2011CB707604) to G.H. is gratefully acknowledged. We thank Professor Neumann of GSI for providing the single ion irradiated PET samples.

\section{REFERENCES}

(1) Kasianowicz, J. J.; Brandin, E.; Branton, D.; Deamer, D. W. Proc. Natl. Acad. Sci. U.S.A. 1996, 93, 13770-13773.

(2) Meller, A.; Nivon, L.; Branton, D. Phys. Rev. Lett. 2001, 86, 3435-3438.

(3) Li, J.; Gershow, M.; Stein, D.; Brandin, E.; Golovchenko, J. A. Nat. Mater. 2003, 2, 611-615.

(4) Kim, Y. R.; Min, J.; Lee, I. H.; Kim, S.; Kim, A. G.; Kim, K.; Namkoong, K.; Ko, C. Biosens. Bioelectron. 2007, 22, 2926-2931.

(5) Moretti, M.; Di Fabrizio, E.; Cabrini, S.; Musetti, R.; De Angelis, F.; Firrao, G. Biosens. Bioelectron. 2008, 24, 141-147.

(6) Kowalczyk, S. W.; Hall, A. R.; Dekker, C. Nano Lett. 2010, 10, 324-328.

(7) Dekker, C. Nat. Nanotechnol. 2007, 2, 209-215.

(8) Healy, K.; Schiedt, B.; Morrison, A. P. Nanomedicine 2007, 2, 875-897.

(9) Miedema, H.; Vrouenraets, M.; Wierenga, J.; Meijberg, W. Nano Lett. 2007, 7, 2886-2891.

(10) Sexton, L. T.; Horne, L. P.; Martin, C. R. Mol. BioSyst. 2007, 3, 667-685.

(11) Siwy, Z. S.; Howorka, S. Chem. Soc. Rev. 2010, 39, 1115-1132.

(12) Lev, A. A.; Korcheve, Y. E.; Rostovtseva, T. K.; Bashford, C. L.; Edmonds, D. T.; Pasternak, C. A. Proc. R. Soc. London, B 1993, 252, 187-192.

(13) Korchev, Y. E.; Bashford, C. L.; Alder, G. M.; Apel, P. Y.; Edmonds, D. T.; Lev, A. A.; Kandi, K.; Zima, A. V.; Pasternak, C. A. FASEB J. 1997, 11, 600-608.

(14) Tabard-Cossa, V.; Trivedi, D.; Wiggin, M.; Jetha, N. N.; Marziali, A. Nanotechnology 2007, 18, No. 305505.

(15) Smeets, R. M. M.; Keyser, U. F.; Dekker, N. H.; Dekker, C. Proc. Natl. Acad. Sci. U.S.A. 2008, 105, 417-421.

(16) Siwy, Z. S. Adv. Funct. Mater. 2006, 16, 735-746.

(17) Siwy, Z. S.; Powell, M. R.; Petrov, A.; Kalman, E.; Trautmann, C.; Eisenberg, R. S. Nano Lett. 2006, 6, 1729-1734.

(18) Powell, M. R.; Sullivan, M.; Vlassiouk, I.; Constantin, D.; Sudre, O.; Martens, C. C.; Eisenberg, R. S.; Siwy, Z. S. Nat. Nanotechnol. 2008, 3, 51-57.

(19) Innes, L.; Powell, M. R.; Vlassiouk, I.; Martens, C.; Siwy, Z. S. J. Phys. Chem. C 2010, 114, 8126-8134.

(20) Apel, P. Y.; Korvhev, Y. E.; Siwy, Z. S.; Spohr, R.; Yoshida, M. Nucl. Instrum. Methods Phys. Res. B 2001, 184, 337-346.

(21) Oganessian, V. R.; Trofimov, V. V.; Vetter, J.; Danziger, M.; Dorschel, B.; Hermsdorf, D. Nucl. Instrum. Methods Phys. Res. B 2003, 208, 166-169.

(22) Wang, X. W.; Xue, J. M.; Wang, L.; Guo, W.; Zhang, W. M.; Wang, Y. G.; Liu, Q.; Ji, H.; Ouyang, Q. J. Phys. D: Appl. Phys. 2007, 40, 7077-7084. 